\section{Gastrointestinal contamination}

\section{To the editor:}

Drummond and colleagues' case report of delayed salicylate toxicity following enteric-coated acetylsalicylic acid (ECASA) overdose $^{1}$ raises the issue of gastrointestinal decontamination - an issue only briefly discussed by the authors. The patient allegedly ingested $30 \mathrm{~g}$ of ECASA 2 hours before she arrived at the emergency department (ED). Her ASA level was $0.3 \mathrm{mmol} / \mathrm{L}$. She underwent gastric lavage with $4 \mathrm{~L}$ of distilled water; no pill fragments were returned, and she was administered $100 \mathrm{~g}$ of activated charcoal.

Is it enough? Considering the evolution of the patient, we can definitely say No.

What gastrointestinal decontamination should have been done? There is no definite evidence-based answer. This was illustrated by a recent telephone survey ${ }^{2}$ to characterize poison control centre recommendations for the gastrointestinal decontamination of a hypothetical patient (ingestion of $32.5 \mathrm{~g}$ of ECASA 1 hour prior to presentation, with a nondetectable ASA level) — a case quite similar to the one in Drummond and colleagues' article. Interestingly, the most frequent action, recommended by 7 of 75 centres, was lavage followed by activated charcoal, with further doses of charcoal if salicylate levels were rising; this was the action taken by Drummond and colleagues.

As discussed by Hoffman, ${ }^{3}$ despite the absence of a definite evidence-based answer, the most informed decision should have been a more aggressive gastrointestinal decontamination. This is based mainly on the fact that a lifethreatening ingestion has occurred and

For reasons of space, letters may be edited for brevity and clarity. that $100 \mathrm{~g}$ of activated charcoal is unlikely to bind $30 \mathrm{~g}$ of salicylate (because of saturation of activated charcoal). What would have been the best available option? Either 1) a dose of activated charcoal followed by whole bowel irrigation (WBI) or 2) repeated doses of activated charcoal until black stools were obtained both with or without prior gastric lavage.

Both options have their problems. WBI is not easy to perform in the ED and could favour dissolution of a bezoar if done improperly. Furthermore, no study assures its efficacy. Clinical experience with iron overdose and volunteer studies suggest that clear effluent does not guarantee clear bowel. Despite the fact that repeated doses of activated charcoal are simpler to administer and could help if there is dissolution of bezoar, the delay of administration could theoretically decrease its efficacy if the ECASA is further down in the gut. Personally, I would have favoured the latter option without gastric lavage.

Drummond and colleagues concluded that for patients with significant ingestion, initial therapy should be considered regardless of salicylate levels. This will not be useful unless significant symptoms or levels of salicylate are present. ${ }^{4}$ The administration of bicarbonates serves 2 goals: urine and blood alkalinization. The goal of urine alkalinization is to increase salicylate excretion by ion trapping. By increasing the urine $\mathrm{pH}$ (only achieved if serum potassium is at the upper limit of normal), more deionized salicylate is present in the urine, thus increasing salicylate excretion. Blood alkalinization will also be useful because it decreases salicylate tissue penetration (mainly central nervous system and heart) by the ssame mechanism. Thus, significant symptoms or levels are required before bicarbonates should be considered. The usefulness of bicarbonates as preventive therapy is not recog- nized and could give a false sense of security if appropriate gastrointestinal decontamination is not performed.

Benoit Bailey, MD, MSc, FRCPC

Divisions of Emergency Medicine and of

Clinical Pharmacology and Toxicology

Department of Pediatrics

Hôpital Ste-Justine

Montreal, Que.

baileyb@total.net

\section{References}

1. Drummond R, Kadri N, St-Cyr J. Delayed salicylate toxicity following enteric-coated acetylsalicylic acid overdose: a case report and review of the literature. CJEM 2001;3 (1):44-6.

2. Juurlink DN, McGuigan MA. Gastrointestinal decontamination for enteric-coated aspirin overdose: what to do depends on who you ask. J Toxicol Clin Toxicol 2000; 38(5):465-70.

3. Hoffman RS. Does consensus equal correctness? [editorial]. J Toxicol Clin Toxicol 2001;38:689-90.

4. Wax PM. Sodium bicarbonate. In: Goldfrank LR, Flomenbaum NE, Lewin NA, Weisman RS, Howland MA, Hoffman RS, editors. Goldfrank's toxicologic emergencies. Philadelphia: Appleton and Lange; 1998. p. 585-6.

\section{An apology to Dr. Cheng}

CJEM wishes to apologize to Dr. Vince T. Cheng for not acknowledging him as the creator of the cartoons featured on pages 33 and 56 of the January 2001 issue of CJEM. "Urban Trauma" is a comic strip centred on the odd events and mishaps of a metropolitan emergency department located in the fictional "East General Hospital." The focus of the humour typically revolves around emergency medical scenarios (medicine, nursing, respiratory therapy), but occasionally Dr. Cheng examines other areas such as hospital clerks, prehospital care or other specialties.

Dr. Cheng is a physician at Mount Sinai Hospital in Toronto. - Eds. 